\title{
Survey of animal bite injuries and their management for an estimate of human
}

rabies deaths in N'Djaména, Chad

Frey $\mathrm{J}^{1}$, Mindekem $\mathrm{R}^{2}$, Kessely $\mathrm{H}^{2}$, Doumagoum Moto $\mathrm{D}^{2}$, Naïssengar $\mathrm{S}^{3}$, Zinsstag $\mathrm{J}^{1,4}$, Schelling $E^{1,4}$

${ }^{1}$ Swiss Tropical and Public Health Institute, Basel, Switzerland, Johanna Frey

(johanna.frey@stud.unibas.ch), Jakob Zinsstag (jakob.zinsstag@unibas.ch), Esther

Schelling (esther.schelling@unibas.ch)

${ }^{2}$ Centre de Support en Santé Internationale, N'Djaména, Chad, Rolande Mindekem

(mrola2002@yahoo.fr), Daugla Doumagoum Moto (daugla.doumagoum@gmail.com), Hamit Kessely (hkessely@yahoo.fr)

${ }^{3}$ Laboratoire de Recherches Vétérinaires et Zootechniques de Farcha, N’Djaména, Chad, Service Naissengar (naissengar@yahoo.fr)

${ }^{4}$ Faculty of Science, University of Basel, Switzerland

Running title: Animal bite rabies study N'Djaména, Chad

Keywords Rabies, N'Djaména, animal bites, post-exposure prophylaxis, wound management, human rabies incidence, human deaths

Word count of body text including acknowledgements (if all changes were accepted): 3425 


\section{Professional qualifications of authors and contributions to the paper}

Johanna Frey (Medical doctor), design of the study, field data collection and writing of the paper.

Jakob Zinsstag, (Veterinary epidemiologist, DVM PhD), design of the study, supervision of data analysis and writing of the paper.

Esther Schelling (Veterinary epidemiologist DVM PhD), design of the study, supervision of the field work, data analysis and writing of the paper, corresponding author.

(esther.schelling@unibas.ch)

Rolande Mindekem (Health geographer, MSc) coordinator of field data collection, contact with authorities, drafting of the paper.

Doumagoum Moto (Medical doctor, MD), medical responsibility of the study, contact with authorities, supervision of field work, drafting of the paper

Hamit Kessely (Geographer, MSc) field data collection, data management, drafting of the paper

Service Naissengar (Biologist), head of the Chadian rabies laboratory, technical support, field data collection, drafting of the paper. 


\section{Summary}

Previous research in N'Djaména, the capital of Chad, showed endemic stability of rabies and that mass dog vaccination over two years interrupts rabies transmission between dogs in the city. The community effectiveness deriving from mass dog vaccination is best shown by a decrease in human rabies deaths. However, no human rabies cases were officially reported for N'Djaména. We conducted a survey of animal bites, with methods previously validated in Tanzania, to estimate the annual human rabies incidence as a baseline prior to mass dog vaccination campaigns. Ninety health care providers were enrolled, representing approximately $50 \%$ of all health care providers in N'Djaména. Data were collected from 557 animal bite victims between September 2008 and April 2009. The probability of dying of rabies after being bitten by a suspected rabid animal was related to the bite location and the probability of receiving post-exposure vaccination. Among 86 people exposed to a suspected rabid animal, $50 \%$ received post-exposure vaccination and further $8 \%$ had their wound cleaned. We estimated annual incidence of bites from suspected rabid animals of $12.9 / 100,000$ and an incidence of 0.7 human rabies deaths/100,000, resulting in a total of seven estimated deaths (95\% confidence interval 4 - 10 deaths) per year in N'Djaména. This is a conservative estimate, since we did not cover all health care providers. In our survey, $14 \%$ of bite victims sought help from veterinarians. Closer cooperation between physicians and veterinarians warrants more effective rabies control. The high proportion $(42 \%)$ of potentially exposed people without post-exposure vaccination or wound treatment necessitates urgent attention. 


\section{Introduction}

Rabies remains an important zoonosis in many developing countries - an estimated 55,000 human deaths occur each year (Knobel et al. 2005). Most human rabies cases that result from rabid dog bites are mainly reported in Asian countries (e.g. Indonesia and India); however, in African countries there is a vast underreporting of rabies in dogs (Kitala et al. 2000) and in humans (Cleaveland et al. 2002).

A person bitten by a rabid animal in the absence of post-exposure prophylaxis (PEP) can develop clinical disease, which is fatal. Providing PEP - repeated injections of rabies vaccine and immunoglobulines to the animal bite victim as soon as possible after exposure, combined with local treatment of the wound (immediate mechanical cleaning of the wound with water and soap followed by disinfection of the wound) - can prevent the development of rabies (WHO 2010). However, PEP, especially the immunoglobulines, is expensive and often not available in low income countries.

In N'Djaména, the capital city of the Chad, the annual incidence risk of rabies in dogs in 2002 was 1.4 per 1000 unvaccinated dogs. One rabid dog or cat exposed 2.3 persons on average, whereby dogs accounted for $95 \%$ of exposures (Kayali et al. 2003). Zinsstag et al (2009) have used a dog-human mathematical transmission model combined with an economic assessment (based on previously collected data during surveys in N'Djaména). They showed that the most cost-effective intervention against dog rabies is to combine parenteral dog vaccination campaigns and PEP, which leads to a break-even in costs after five years when compared to costs of PEP alone.

To date, human rabies deaths have never been estimated in N'Djaména. We found three reported human rabies cases in Chad in the RABNET database (hosted by WHO) - and they were all for 1997. In Chad there is currently no capacity for human post-mortem rabies diagnosis by immunofluorescence. 
Clinical human rabies cases can be misdiagnosed. In Malawi, among 26 fatal cases initially assigned to cerebral malaria, three were diagnosed with rabies during post-mortem examinations, and 14 of 133 fatal cases assigned to central nervous signs were in fact deaths due to rabies (Mallewa et al. 2007).

Cleaveland et al. (2002) found that active detection of human rabies deaths is difficult due to low incidence and the need to set-up specific detection studies such as collection of verbal autopsy data from household surveys. Passive surveillance may be insufficient, leading to vast underreporting of human rabies cases. However, animal bites can be surveyed given their rather high incidence and the preference of victims to seek professional care. Cleaveland et al. (2002) used a probability decision tree to estimate human mortality from information provided by animal bite victims. After validation of the approach in field studies, they showed that, in rural Tanzania, the true incidence of human rabies was 10 to 100 times higher than the reported incidence.

In view of future dog rabies elimination in N'Djaména, the effectiveness of the mass dog vaccination should show a reduction in human exposure to suspected rabid animals. However human clinical rabies in N'Djaména can only be estimated. This paper aims to adapt the probability tree model, inferring humand rabies death from animal bites, proposed by Cleaveland et al. (2002) to the urban setting of N'Djaména, the capital of Chad. In this way we attempt to estimate indirectly human rabies incidence from an animal bite survey in health facilities in N'Djaména and to describe the treatments that animal bite victims have received.

\section{Materials and Methods}

\section{Collaborating health care providers}

Health care providers for animal bite victims 10 municipal city subdivisions (Arrondissements) of N'Djaména were contacted and registered as: i) hospitals, treatment and health centres; ii) health clinics; iii) pharmacies and pharmaceutical shops and iv) 
veterinary clinics. Bite victims sought counselling and first aid treatment at any of these health care providers. We have not differentiated between private and public health centres / clinics. Our enrolment of private health care providers (clinics, pharmacies and pharmaceutical shops) was compared to authorised private health centres / clinics and pharmacies in N'Djaména (Gami 2008) and of public health providers to operational public hospitals and health centres reported in the Health Statistical Report of 2008 (Ministère de la Santé Publique du Tchad 2008).

The heads of 10 municipal Arrondissements were visited in order to inform them on the study and with the request to distribute the information to their heads of districts.

\section{Data collection}

We developed a data sheet (in French) to collect data on the age of a bite victim, site of the bite injury (indicated on drawings of an adult and a child), type of treatment (including postexposure vaccination) and whether the animal was suspected by the bitten person of being rabid and/or being vaccinated. We also asked about wound treatment and whether a bite victim was referred from or to another health care provider. The latter was to avoid double inclusion of a same bite victim. The health care providers completed the information sheet together with the bite victim.

From September 2008 to April 2009, all collaborating health care providers were visited every second week to collect the forms. In the same period, all dogs put under observation at the veterinary clinic of N'Djaména were recorded. If the suspected rabid animal died or showed signs of rabies (the latter were euthanized), the brain tissue of the animal was tested at the Laboratoire de Recherches Vétérinaires et Zootechniques de Farcha (LRVZ) with the direct fluorescent antibody test - the WHO-recommended gold standard of rabies diagnosis (World Health Organization 2005; Dean et al. 1996).

\section{Ethical considerations}


We obtained ethical clearance from the Ethics Committee of Basel (EKBB) and the study was authorized by the Chadian Ministry of Health. Adapted information material (leaflets and posters) for animal bite victims (Durr et al. 2009) was handed out to bite victims. The information included correct management of a bite wound, a contact number of a physician and the information that post-exposure vaccination is available for free at the Centre de Support en Santé Internationale, where a stock of rabies and tetanus vaccines was stored for the study. Note that no anti-rabies immunoglobulines were available in Chad.

\section{Data analyses}

The data was entered into Microsoft Access and analysed in Stata 10 (StatCorp LP). Age was categorised regarding age-dependent treatment procedures, and height at age, in adults ( $\geq 13$ years) and children (<13 years). The sites of the reported bite injuries were categorised as i) head (head, face and neck); ii) arm (hands, arms and shoulders); iii) trunk; and iv) legs. When more than one bite location was registered, the site with the higher probability of developing rabies (head or upper extremity) was retained (Hattwick \& Gregg 1975). The reported severity of the wound was classified as i) deep wound; ii) lacerated wound; iii) superficial wound; and iv) scratch. We considered animals as vaccinated against rabies if the reported vaccination took place less than 12 months prior to the bite. The chi square test (Fisher's exact test where appropriate) was used to assess possible associations between age, site of bite, severity of injury and sex of bite victims.

To estimate the incidence of human cases of rabies, we have used the decision tree designed by Cleaveland et al. (2002). The starting point was $\mathrm{P} 1$, the probability of a suspected animal being rabid. P2 - P5 were defined by the proportions of bite injury sites. P6 - P9 were the probabilities of developing rabies after a bite from a rabid animal depending on its location. P10 was the probability of receiving post-exposure vaccination (Table 5). Finally, the probability of dying of rabies (Pdeath) after being bitten by a rabid suspect animal was calculated as follows: Pdeath $=\mathrm{P} 1 \times[(\mathrm{P} 2 \times \mathrm{P} 6)+(\mathrm{P} 3 \times \mathrm{P} 7)+(\mathrm{P} 4 \times \mathrm{P} 8)+(\mathrm{P} 5 \times \mathrm{P} 9)] \times(1-$ 
P10). Pdeath considered that a proportion of people bitten by a suspect rabid animal had received post-exposure vaccination and thus would not develop rabies.

The total number of deaths due to rabies per year was calculated as Ndeath $=(\mathrm{i} x \mathrm{Q} x$ Pdeath)/100,000, where i was the incidence of suspected animal bites per year per 100,000 inhabitants, and $Q$ the total population at risk. The population at risk $Q$ was taken from the census of 2009 as $Q=993,447$ people (Ministère de l'Economie et du Plan 2009). The annual incidence i of suspected animal bites was calculated from the observed proportion of suspected bites (psuspected) multiplied with the number of suspected animal bites (nbites) during eight months and adjusted to one year and 100,000 people at risk as given below.

$\mathrm{i}=$ psuspected * nbites * 12 months * $100,000 /(8$ months * Q)

We have taken a binomial distribution for P2 - P5 and a Pert distribution for P1 and P6 - P9 (Table 5). The Pert distribution is often used on parameters that have a shaped-like normal distribution but with defined upper and lower limits. This is the case for example with expert opinions (most likely is the mode and is within minimum and maximum values) (Malcolm et al. 1959). The number of suspected animal bites bbite was expressed as binomial distribution (Table 5). Pdeath and Ndeath were estimated by Monte Carlo simulations with 10,000 iterations using "Ersatz" software (EpiGear International Pty Ltd Brisbane, Australia, 2009-2012, www.epigear.com).

\section{Results}

Ninety public and private health care providers in N'Djaména were contacted and all agreed to be enrolled in the study. Because bite victims sometimes contacted a veterinarian, we also included five veterinary clinics in the survey. These received 77 of the registered 557 bite victims (Table 1). In 2008, a study established in parallel to our survey a list with private health centres/clinics and pharmacies in N'Djaména. Relating to Gami (2008), 50 private treatment/health centres and clinics were operational in N'Djaména, whereby we have enrolled 12 private health centres and 19 private health clinics, which represent $62 \%$ of 
private health care structures. There were 28 pharmacies and 71 pharmaceutical stores, whereof we have contacted 17 pharmacies (61\%) and 10 pharmaceutical stores $(14 \%)$. In addition, there were 38 public health structures (six hospitals and 32 health centres) (Ministère de la Santé Publique du Tchad 2008), of which we have enrolled 27 (71\%). Overall, we have enrolled approximately $50 \%$ of the public and private health care providers.

We collected a total of 557 completed data sheets from 62 health care providers (Table 1). Two bite victims were registered twice and the data of their first visit were excluded from analyses.

The home Arrondissement was known for 528 bite victims. Figure 1 shows the distribution of the health care providers and reported animal bite injuries per 100,000 inhabitants in the 10 Arrondissements of N'Djaména, with the highest rates in Arrondissements 6 and 7. More records per month were collected during the hot, dry season months (March and April), than during the dry season months (September - February) (Figure 2).

As to biting animals, 505 were dogs, 29 cats, 20 monkeys and 1 was a donkey. Among these, 219 dogs (43\%), 3 cats $(10 \%)$, and 12 monkeys $(60 \%)$ were reported to be vaccinated against rabies, and of all these, $62.9 \%$ had been vaccinated within the past 12 months. Another $40.7 \%$ of all animals were not vaccinated and, for $13.6 \%$, the status was unknown. The victims reported that $16 \%$ of dogs, $7 \%$ of cats and $5 \%$ of monkeys were suspected of being rabid ( $n=86$ within the eight months of the survey). Another $74.9 \%$ of animals were declared non-suspicious and, for $9.7 \%$, no declaration could be recorded. Thirty-nine percent of the suspected dogs were animals unknown to the bite victim but this proportion was lower (12\%) among non-suspected dogs (Table 2). An animal biting a child was significantly more often declared suspicious than when biting an adult (21.2 vs. 14.3\%). Reports on further bite victims were significantly more frequent for suspect dogs than for non-suspects $(49 \%$ vs. $16 \%)$ and the mean number of bite victims was greater (2.1 vs. 1.3 people). 
During the study period, 27 suspected rabid animal carcasses were brought for examination to the "Laboratoire de Recherches Vétérinaires et Zootechniques de Farcha". The 23 dogs had bitten 37 persons (an average of 1.6 persons) and the four cats 6 persons (an average of 1.5 persons). Bite victims of $75 \%$ of these suspected animals had been registered as a bite victim in this study. Thirteen carcasses were confirmed rabid (12 dogs, 1 cat), four tested negative and 11 could not be tested due to decomposed brain samples. Of all the tested samples, $76 \%(95 \% \mathrm{Cl} 50-93 \%)$ were confirmed rabid ( $86 \%$ of dogs, $50 \%$ of cats).

Among the bite victims, $38 \%$ were children (37\% girls, $63 \%$ boys) and $62 \%$ adults $(42 \%$ women, $58 \%$ men). The median age was 18 years, with a range between 2 months and 79 years. All bites to the head were in children. The bite location differed significantly between children and adults (with children being rather bitten in the upper body part), but not between males and females (the higher frequency of male bite victims is reflected in all bite locations). We did not see significant differences between the reported severity of the bite and the locations (Table 3).

One third of the bite victims (182 of 550 with data) went to a health care provider the same day as they were bitten (with a range of 0 to 120 days and a median of 1 day). Fifty percent of the 86 people bitten by a suspected rabid animal received post-exposure vaccination (and among the 43 vaccinated, 1 person had also cleaning of the wound at the health care provider). These proportions were significantly lower among people bitten by a nonsuspected animal (Table 4).

\section{Incidence of human cases of rabies}

With Monte Carlo simulation we derived a probability of dying of rabies after being bitten by a suspected rabid animal [Pdeath] of $5.3 \%(95 \% \mathrm{Cl} 3.2-7.8 \%)$ in the city of N'Djaména.

The annual incidence of bites from suspected rabid animals was derived from the 86 people bitten by a suspect dog within 8 months. The probability of being bitten leading to an incidence of $12.9 / 100,000$ over 12 months. Note that we have recorded bites from suspected 
victims in both the dry and rainy season in the 8 months of recording. The annual total number of human rabies deaths [Ndeath] in N'Djaména was calculated as 6.6, thus 7 deaths $(95 \% \mathrm{Cl} 3.9-10.4)$ or, expressed as annual human rabies incidence, 0.7 per $100,000(95 \%$ Cl $0.4-1.05)$.

A sensitivity analysis of the stochastic model calculated Spearmans rank correlation coefficients $(\mathrm{RCC})$ for all parameters. The probability of death and the number of death were most sensitive $(\mathrm{RCC}>0.35)$ to the probability developing rabies from an arm injury, the probability of receiving PEP and the probability of being bitten to the arm.

\section{Discussion}

Ninty-seven percent of bite victims of a suspect rabid animal in N'Djaména were exposed to a dog, which is in agreement with observations in other cities (Wandeler et al. 1993) and previous studies in N'Djaména (Kayali et al. 2003). Most of the bite reports were from Arrondissement 6 and 7, which matches with observations by Mindekem (2003), who reported that Arrondissement 6 and 7 had higher dog/person ratios compared to the others.

Several health care providers have stated that more animal bites are seen during the hottest months, because animals are irritated by the heat and as a consequence bite more easily. Indeed, we received more forms of bite victims in March and April (the very hot months), however, the proportion of rabid suspected bites remained constant over the 8 surveyed months.

Animals were more often classified as suspect when they bit to the head or the upper extremity. Consistent with other studies, likely due to their height and their more careless approaching of animals, children were bitten twice as often in dangerous locations (head, shoulders or arms) than adults, and thus were at higher risk of being infected and dying of rabies, because the probability of developing rabies after a bite to the head or to the arms is highest (Hattwick \& Gregg 1975). 
In our urban study, $16 \%$ of all dogs inflicting bite injuries were suspect of being rabid, which is much lower than the $77 \%$ found in rural Uganda (Fevre et al. 2005) and the $75 \%$ in rural Tanzania (Cleaveland et al. 2002) - although only 35\% of the owned dogs in our study were vaccinated. It seems more likely in an urban than a rural setting that bite victims, also those without suspected rabies exposure, seek health care (we cannot further quantify the proportions of all animal bite victims seeking professional help).

We compared our results to the incidence estimate of 1.4 per 1000 dogs per year and 2.3 exposed persons per animal by Kayali et al. (2003) using a total dog population estimate of 23,500 dogs in N'Djaména in 2001 (Mindekem et al. 2005). Thus, 75 persons per year were exposed to a suspected rabid animal in N'Djaména, resulting in an incidence of death (while considering that $50 \%$ were protected by post-exposure vaccination) of $0.5 / 100,000$ (for a human population of 770,000 in 2002). The estimated human death incidence from our animal bite study $(0.64 / 100,000)$ is 1.4 times higher than that calculated from the dog rabies incidence in 2001.

No human rabies cases are reported in Chadian official health records and we thus cannot calculate an under-reporting of human rabies cases in N'Djaména. Our indirect estimate of human rabies incidence indicates that human rabies cases likely occur N'Djamena and is in conflict with the fact no human rabies are reported in official health records. Given the endemic stability of rabies in N'Djaména (Zinsstag et al. 2009), we extrapolated our data to an estimate of an annual exposure incidence. Our estimate of the proportion of deaths among exposed people is comparable to Cleaveland et al. (2002) $(5.3 \%$ in this study and $4.4 \%$ in Tanzania), reflecting only slight differences in bite localizations.

The coverage of enrolled health care providers in our study was approximately $50 \%$. Therefore, we assume that the true number of people exposed to suspected animals could be higher (i.e. double) than that recorded, but the effective coverage of health care providers is not known. Among the bite victims of animals brought for rabies examination to the national veterinary laboratory during the same study period, we have registered $75 \%$ at a 
health care provider. Assuming that our study covered $75 \%$ of the effective bite victims, human rabies mortality could be as high as $0.93 / 100,000$. However, we also saw that $32 \%$ of rabid suspect animals were animals not known to the bite victim (and their ownership status was not known). For comparison, Kayali et al. (2005) found that $85 \%$ of the rabid dogs were owned. We thus likely have underestimated the exposure frequency, but the reported 86 bites from suspected animals among the 557 bite victims were underestimated to a lesser extent. We conclude that the estimated seven human rabies deaths per year in N'Djaména is a conservative estimate.

Only $50 \%$ of the victims of suspected rabid animal bites received post-exposure vaccination. In Chad, as in many developing countries, repeated vaccinations are given as sole treatment and not combined with injections of anti-rabies-virus-immunoglobulines. Post-exposure vaccinations often remain unavailable and are expensive (approximately 16 USD / vaccine, five vaccines needed). Where post-exposure vaccination is not available, it is even more crucial to clean the wound properly, but of those potentially exposed and who did not receive vaccinations (43 victims), only seven had their wound cleaned by the health care provider, which requires urgent attention.

To note are the $14 \%$ of bite victims who have first sought health care at a veterinary clinic. We found in this group that a higher proportion of victims being bitten by a rabid suspected animal received post-exposure vaccination than among other bite victims ( $62 \%$ vs $50 \%)$. This might be due to the fact that the human vaccine was available at the largest veterinary clinic in N'Djaména, but also due to a better information about rabies of veterinary than of medical staff.

\section{Conclusions}

This study suspects the presence of human rabies cases in N'Djaména based on an animal bite survey. There were only officially reported rabies cases for animals, but not for people. This is seen in several Sahelian cities. New approaches to estimate the human rabies 
burden should use the available information on rabies in animals. This survey will also serve as a baseline to show if the government-funded mass dog rabies vaccination campaign, which has started in 2012, will have a measurable effect on communities in terms of lower animal bite frequency, post-exposure vaccinations and human rabies death incidences. Closer data exchange between health and veterinary clinics would benefit from a fuller description of the status of rabies in Chad. Given the estimate of seven human deaths due to rabies each year in N'Djaména, and the broad fear of rabies in the population, mass dog vaccination campaigns covering the whole city are warranted.

\section{Acknowledgments}

Our great appreciation goes to the health care providers and the animal bite victims in N'Djaména, for their active participation in our study. We thank the Swiss Federal Veterinary Office for the financial support. Finally we would like to thank Prof. Christoph Hatz and Dr. Kaspar Wyss for their mentoring of the medical aspects. 


\section{References}

Cleaveland S, Fevre EM, Kaare M, \& Coleman PG (2002) Estimating human rabies mortality in the United Republic of Tanzania from dog bite injuries. Bull. World Health Organ 80, 304-310.

Dean DJ, Abelseth MK, \& Atanasiu P (1996) The fluorescent antibody test. In Laboratory Techniques in Rabies. 4th edition edn. (eds. FX Meslin, MM Kaplan, \& H Kaprowski) World Health Organization, Geneva.

Durr S, Mindekem R, Kaninga $\mathrm{Y}$ et al. (2009) Effectiveness of dog rabies vaccination programmes: comparison of owner-charged and free vaccination campaigns. Epidemiol. Infect. 137, 1558-1567.

Fevre EM, Kaboyo RW, Persson V et al. (2005) The epidemiology of animal bite injuries in Uganda and projections of the burden of rabies. Trop Med Int. Health 10, 790-798.

Gami, J. P. L'offre de soins modernes à but lucratif dans la ville de N'Djaména, Tchad. Rapport de recherche, 1-39. 2008. Université Paris Ouest Nanterre La Défense, Laboratoire Espace Santé Territoires (LEST).

Hattwick MAW \& Gregg MB (1975) The disease in man. In The natural history of rabies. 1 edn. (ed. GM Baer) CRC Press, New York, 281-304.

Kayali U, Mindekem R, Yemadji N et al. (2003) Incidence of canine rabies in N'Djamena, Chad. Prev. Vet. Med. 61, 227-233.

Kitala PM, McDermott JJ, Kyule MN, \& Gathuma JM (2000) Community-based active surveillance for rabies in Machakos District, Kenya. Prev. Vet. Med 44, 73-85.

Knobel DL, Cleaveland S, Coleman PG et al. (2005) Re-evaluating the burden of rabies in Africa and Asia. Bull World Health Organ 83, 360-368.

Malcolm DG, Roseboom CE, Clark CE \& Fazar W (1959) Application of a technique for research and development program evaluation. Operations Research 7, 646-649.

Mallewa M, Fooks AR, Banda D et al. (2007) Rabies encephalitis in malaria-endemic area, Malawi, Africa. Emerg. Infect. Dis. 13, 136-139.

Mindekem, R. The geography of risk factors of rabies transmission in urban settings: the case of N'Djaména, Chad [in French]. 2003. Montpellier, France: Universite' Paul-Valery, U.F.R. III.

Mindekem R, Kayali U, Yemadji N, Ndoutamia AG, \& Zinsstag J (2005) La Démographie Canine Et Son Importance Pour La Transmission De La Rage Humaine à N'Djaména. Médecine Tropicale 1, 5358.

Ministère de l'Economie et du Plan. Deuxième recensement générale de la population et de l'habitat de la République du Tchad, Résultats Globaux. Institut national de la statistique, des études économiques et démographiques INSEED. RGPH2, Septembre 2009. 2009.

Wandeler Al, Matter HC, Kappeler A, \& Budde A (1993) The ecology of dogs and canine rabies: a selective review. Rev. Sci. Tech. 12, 51-71.

WHO. Rabies. Fact Sheet N99. 2010. WHO.

World Health Organization. WHO Expert Consultation on Rabies. 931, 1-121. 2005. Geneva, WHO. WHO Technical Report Series.

Zinsstag J, Durr S, Penny MA et al. (2009) Transmission dynamics and economics of rabies control in dogs and humans in an African city. Proc. Natl. Acad. Sci. U. S. A 106, 14996-15001. 


\section{List of Figures}

Figure 1 The distribution of the health care providers (all enrolled: light grey bars and those with reports: dark grey bars)) and reported animal bite injuries per 100,000 inhabitants (diamonds) in the 10 Arrondissements of N'Djaména

Figure 2 Number of reports received per month (Dark grey: total number; light grey: reports of bites from suspect rabid animals) and average temperatures (dots). 


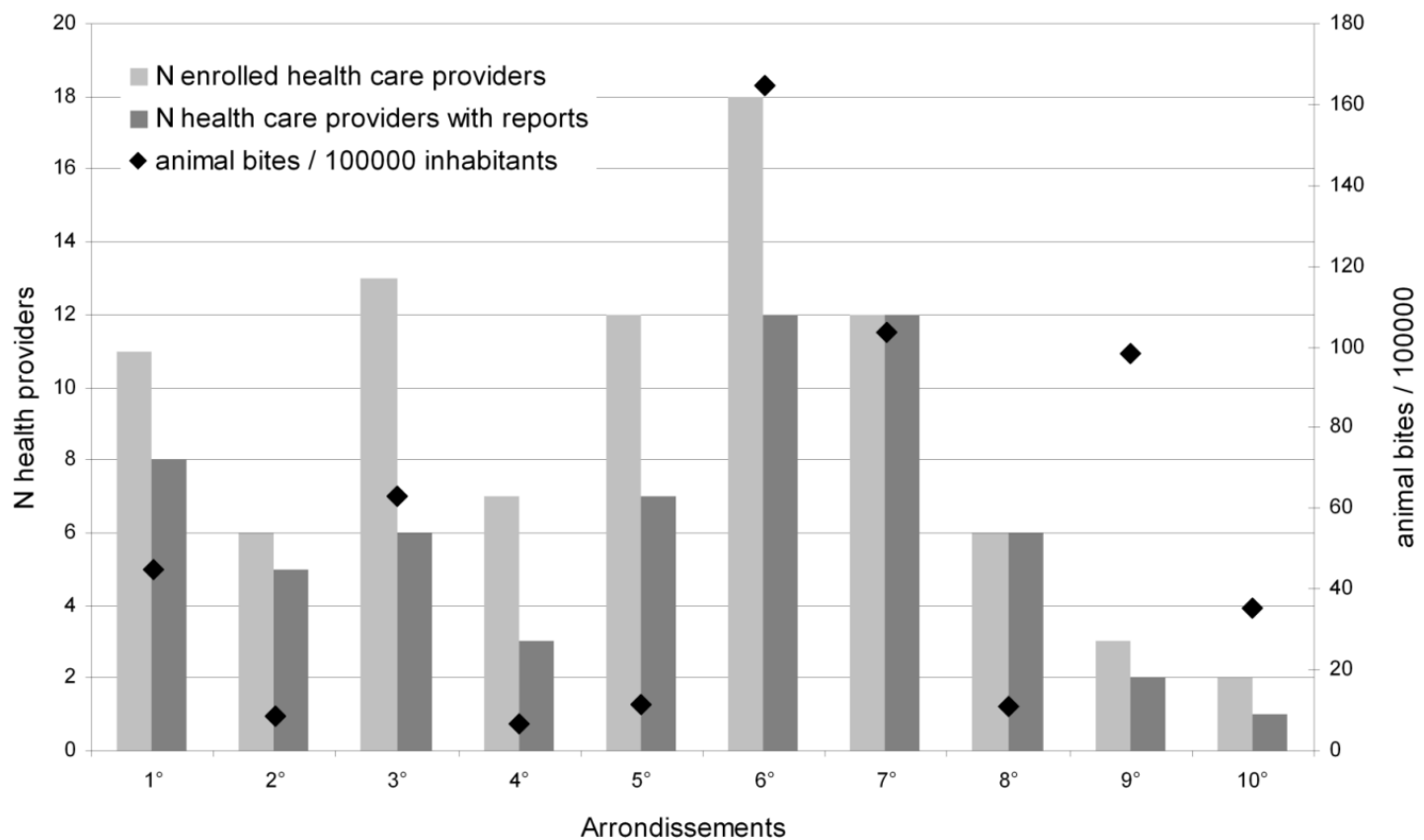




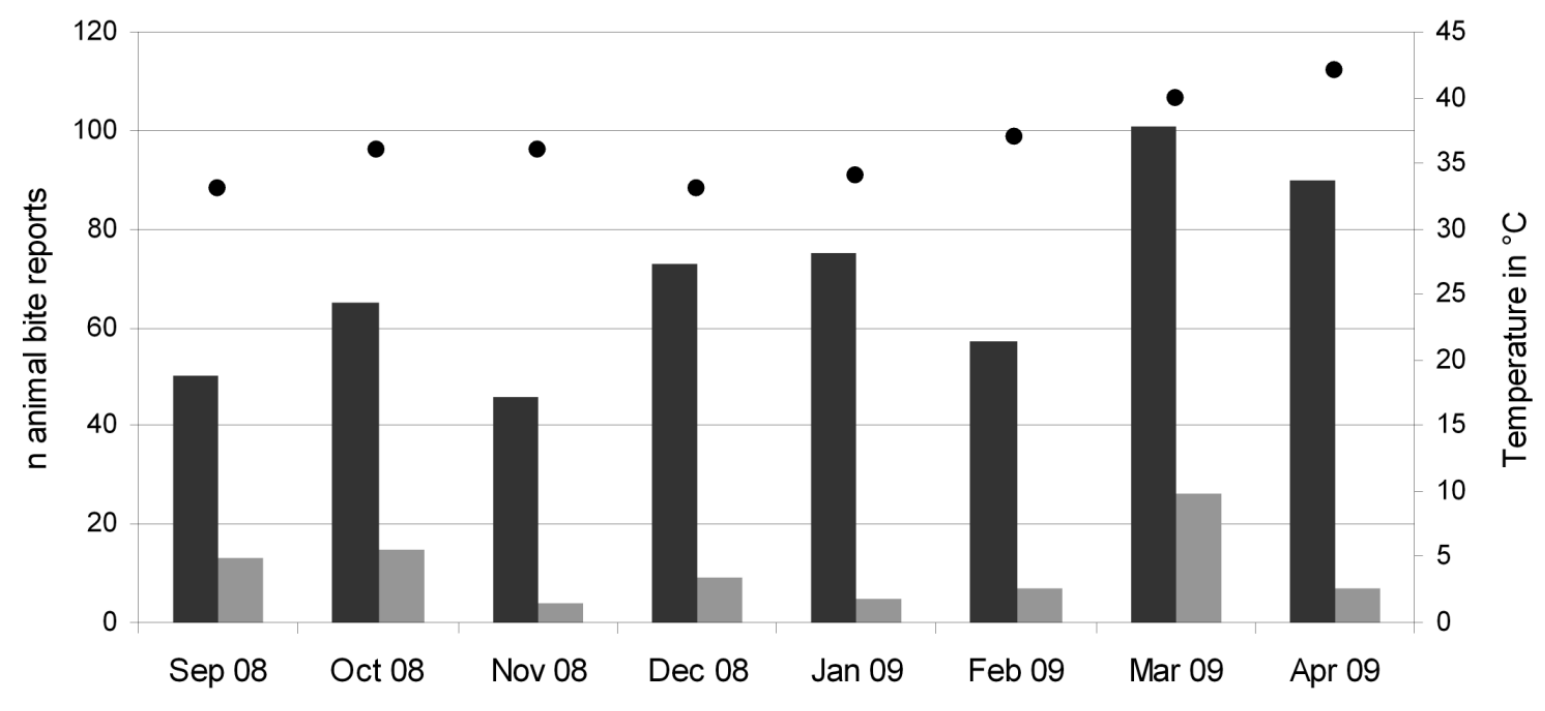

